\title{
Fuzzy Logic Based Dryer Controller
}

\author{
Aye Mar Khin ${ }^{1}$, Aye Myint Thwe' \\ Lecturer $^{1,2}$ \\ ${ }^{12}$ Department of Engineering Physics \\ ${ }^{2}$ University of Technology (Yatanarpon Cyber City) \\ Pwin Oo Lwin, Mandalay Division \\ Myanmar
}

\begin{abstract}
The fuzzy logic control system determines the real world constraint of the dryer's heating rate to the dryer driver circuit which is constructed with the pulse width modulation (PWM) technique. Input and output operations to and from the computer system are carried out via parallel interfacing and Turbo C++ programming. The humidity sensing circuit is constructed around a 555 timer with astable operation which is a non-sinusoidal oscillator. This humidity sensing circuit produces a rectangular wave with the frequency indirectly proportional to the sensed humidity. In order to achieve the perfectively symmetrical square wave as well as to reduce the frequency value, the clocked flip-flop, CD4017BC that is divided by 10 Johnson counter with 10 decoded outputs and a carry out bit is added to the timer's output. The resultant frequency is applied into the computer system integrating with the fuzzy logic and it determines the frequency value and its rate of change which are input to the fuzzy logic control system.
\end{abstract}

Key Words: Fuzzy Logic, CD4017BC, humidity sensor, 555 Timer.

\section{INTRODUCTION}

Fuzzy logic uses approximate instead of exact information, imitating the human thinking. Nowadays fuzzy logic is used in control systems and in decision support systems where the problem description approach can not be precise. A fuzzy system is formed of output and input variables. The mathematics generated by these theories is consistent, and fuzzy logic may be a generalization standard of classic logic [3,5]. Nowadays fuzzy logic is used in control systems and in decision support systems where the problem description approach cannot be precise. Drying Control System with the application of fuzzy control technology, the drying system processes some advantages such as the intellectualization level and the small power [7]. In this system, there are only eight different values for moisture values and its rate of changes. These eight value levels are partitioned into four membership functions and the fuzzy values assigned are limited, only three different fuzzy values, $0,0.5$ and 1 which are limited.

There are five membership functions of the duration of the pulse. Only three different fuzzy values assigned to the nine different durations partitioned with five output membership functions become restricted. When all the possible combinations of different values are calculated, it is found that there are only six different fuzzy outputs. The DC motor applied in this system's prototype has a small toque and cannot start to rotate instantly with the small duration driving pulse.

\section{FUZZY LOGIC THEORY}

Fuzzy logic is a formulation of logic using mathematical framework to deal with concepts such as few, very, much and many. Fuzzy logic can help us make decisions when lack of specific facts or the knowledge is imprecise or far from complete. The fuzzy controller as an artificial decision maker operates in a closed-loop system in real time. Fuzzy controller as shown in Figure 3.1 is composed of the following four elements $[3,4,5]$ :

1. A rule-base (a set of If-Then rules) holds the knowledge, in the form of a set of rules, of how best to control the system.

2. The inference mechanism evaluates which control rules are relevant at the current time and then decides what the input to the plant should be.

3. The fuzzification interface simply modifies the inputs so that they can be interpreted and compared to the rules in the rulebase. 
International Journal of Advances in Scientific Research and Engineering (ijasre), Vol 6 (4), April -2020

4. The defuzzification interface converts the conclusions reached by the inference mechanism into the inputs to the plant [6].

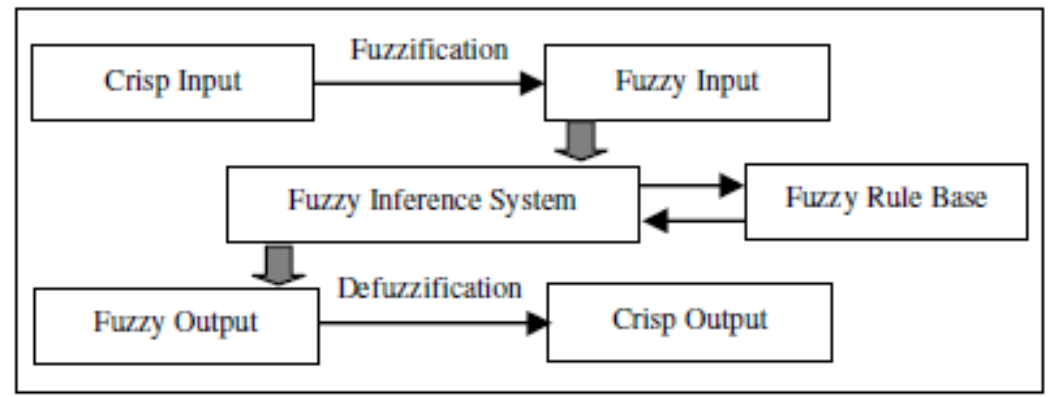

Figure1. General Scheme of Fuzzy Model

\subsection{Fuzzzification}

The choice of the inputs and outputs of the controller is fundamentally important in the remainder of the fuzzy control design process. The fuzzification process act to obtain a value of an input variable and finding the numeric values of the membership functions that are defined for that variable $[3,5]$.

\subsection{Membership Functions}

Fuzzification involves two processes: derive the membership functions for input and output variables and represent them with linguistic variables. This process is equivalent to converting or mapping classical set to fuzzy set to varying degrees. In practice, membership functions can have multiple different types, such as the triangular waveform, trapezoidal waveform, Gaussian waveform, bell-shaped waveform, sigmoidal waveform and S-curve waveform. The exact type depends on the actual applications $[3,5]$.

\subsubsection{Membership Value Assignment for Moisture Value}

To construct the membership functions for current moisture values are partitioned into eight levels to fit for the inputs membership functions. These values are categorized into four membership functions; Very Small (VS), Small (S), Medium (M) and Large (L). The VS membership function defines value 0 has fuzzy value ' 1 ', value 1 has fuzzy value ' 0.5 ' and all other voltages have fuzzy value zeros, and other membership functions as well. All the membership functions categorizing the moisture values with corresponding fuzzy values are summarized in Table 1.

Table1. Membership Values for Moisture Value

\begin{tabular}{|c|c|c|c|c|c|c|c|c|}
\hline \multirow{2}{*}{$\begin{array}{c}\text { Membership } \\
\text { Functions }\end{array}$} & \multicolumn{9}{|c|}{ Moisture Value } \\
\cline { 2 - 10 } & 0 & 1 & 2 & 3 & 4 & 5 & 6 & 7 \\
\hline Very Small (VS) & 1 & 0.5 & 0 & 0 & 0 & 0 & 0 & 0 \\
\hline Small (S) & 0 & 0.5 & 1 & 0.5 & 0 & 0 & 0 & 0 \\
\hline Medium (M) & 0 & 0 & 0 & 0.5 & 1 & 0.5 & 0 & 0 \\
\hline Large (L) & 0 & 0 & 0 & 0 & 0 & 0.5 & 1 & 1 \\
\hline
\end{tabular}

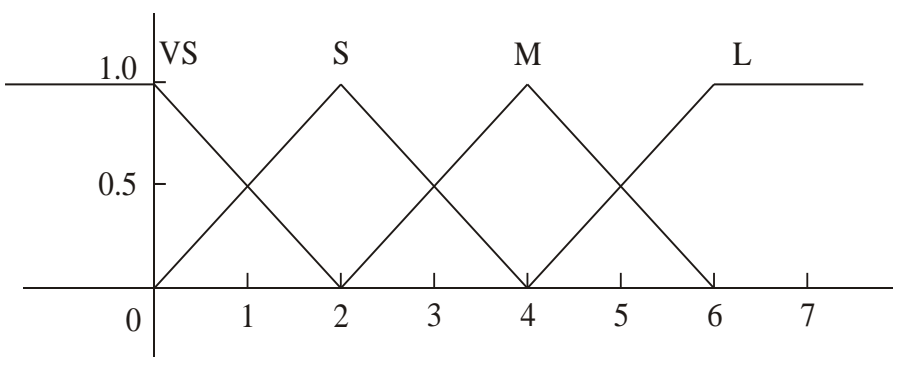

Figure2. Membership Functions of Moisture Value

The membership functions for moisture value are graphically represented in Figure 2. In this graph, the $\mathrm{x}$-axis represents the moisture value and the y-axis represents the corresponding fuzzy values according to the relative membership functions. 
International Journal of Advances in Scientific Research and Engineering (ijasre), Vol 6 (4), April -2020

\subsubsection{Membership Value Assignment for Moisture's Rate of Change}

All the membership functions categorizing moisture's rate of change with corresponding fuzzy values are summarized in Table 2 and graphically represented in Figure 2.

Table2. Membership Values for Moisture's Rate of Change

\begin{tabular}{|c|c|c|c|c|c|c|c|c|}
\hline \multirow{2}{*}{$\begin{array}{c}\text { Membership } \\
\text { Functions }\end{array}$} & \multicolumn{8}{|c|}{ Moisture's Rate of Change } \\
\cline { 2 - 9 } & 0 & 1 & 2 & 3 & 4 & 5 & 6 & 7 \\
\hline Very Small (VS) & 1 & 0.5 & 0 & 0 & 0 & 0 & 0 & 0 \\
\hline Small (S) & 0 & 0.5 & 1 & 0.5 & 0 & 0 & 0 & 0 \\
\hline Medium (M) & 0 & 0 & 0 & 0.5 & 1 & 0.5 & 0 & 0 \\
\hline Large (L) & 0 & 0 & 0 & 0 & 0 & 0.5 & 1 & 1 \\
\hline
\end{tabular}

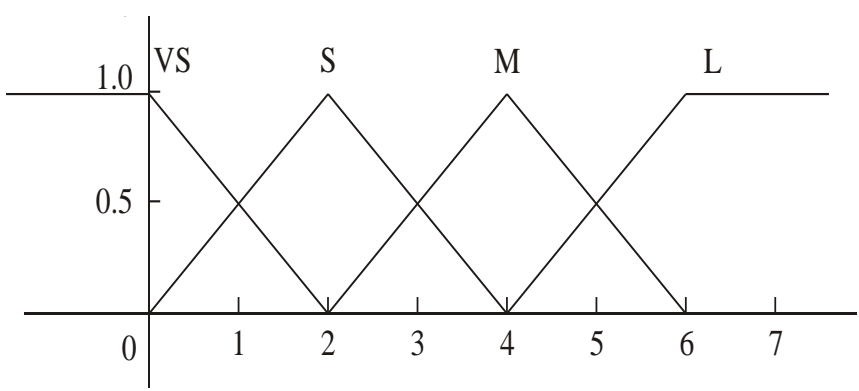

Figure3. Membership Functions of Moisture's Rate of Change

\subsubsection{Membership Value Assignment for Duration of Pulse}

The fuzzy logic outputs determining the width of the pulse have to control the speed of the heater. The membership functions of the durations of the pulse have to categorize the durations for both positive and negative values. All the membership functions for the fuzzy output (duration of pulse) with corresponding fuzzy values are summarized in Table 3 and graphically represented in Figure 4.

Table3. Membership Values for Duration of Pulse

\begin{tabular}{|c|c|c|c|c|c|c|c|c|c|}
\hline \multirow{2}{*}{ S } & \multicolumn{10}{|c|}{ Durations of Pulse } \\
\cline { 2 - 11 } & -4 & -3 & -2 & -1 & 0 & 1 & 2 & 3 & 4 \\
\hline Left Big (LB) & 1 & 0.5 & 0 & 0 & 0 & 0 & 0 & 0 & 0 \\
\hline Left (L) & 0 & 0.5 & 1 & 0.5 & 0 & 0 & 0 & 0 & 0 \\
\hline Zero (Z) & 0 & 0 & 0 & 0.5 & 1 & 0.5 & 0 & 0 & 0 \\
\hline Right (R) & 0 & 0 & 0 & 0 & 0 & 0.5 & 1 & 0.5 & 0 \\
\hline Right Big (RB) & 0 & 0 & 0 & 0 & 0 & 0 & 0 & 0.5 & 1 \\
\hline
\end{tabular}

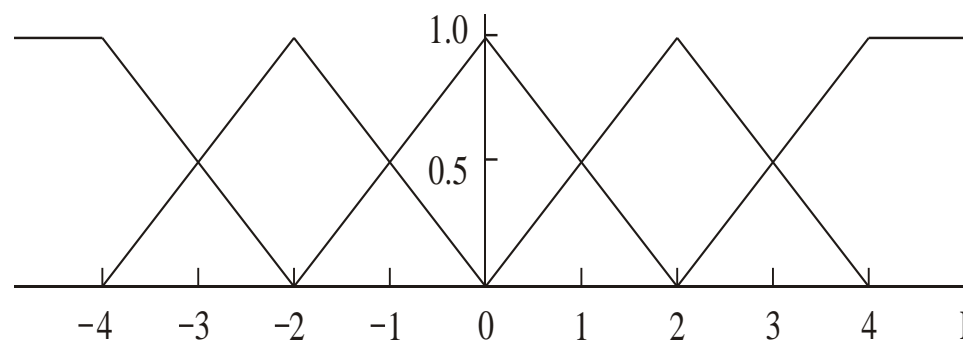

Figure4. Membership Functions of Durations of Pulse 


\subsection{FAM Table of the System}

The fuzzy logic of the system contains two fuzzy inputs (moisture value and its rate of change) and one fuzzy output (duration of the pulse) [4]. The two fuzzy inputs are partitioned into four membership functions and the fuzzy output is partitioned into five membership functions. To fit for this situation, the fuzzy associative memories (FAM) table is designed as in Table 4. The input membership functions are taken as input of the FAM table and the output of the FAM table is partitioned into five regions; Fast Big (FB), Fast (F), Zero (Z), Slow (S) and Slow Big (SB).

Table4. Fuzzy Associative Memories (FAM) Table of the System

\begin{tabular}{|c|c|c|c|c|}
\hline & VS & S & M & L \\
\hline VS & Z & F & FB & FB \\
\hline S & S & Z & F & FB \\
\hline M & SB & S & Z & F \\
\hline L & SB & SB & S & Z \\
\hline
\end{tabular}

\section{IMPLEMENTATION OF THE SYSTEM}

To control the heating rate of the dryer, first of all, it is needed to sense out the amount of humidity. If the amount of humidity increases, the heating rate must also be increased. If the amount of humidity decreases, the heating rate must also be decreased. The relative humidity sensor is applied to sense the amount of humidity. The relative humidity sensor is actually a capacitor and its capacitance changes with the surrounding amount of humidity. The relative humidity is used as the timing capacitor in the 555 timer constructed as astable operation. As stated before, the 555 timer's astable operation serves as a square wave clock generator and in this system; it is termed as humidity sensing circuit.

The output frequency of the humidity sensing circuit is a square wave clock of relatively high frequency of few thousands cycles per second. To get perfectively symmetrical square wave as well as to reduce the frequency in order to be suited to be applied into the computer system, the output frequency of the humidity sensing circuit is divided by means of flip-flops' frequency division property and the divided frequency representing the amount of humidity is applied into the computer system. The amount of humidity and the rate of change of the amount of humidity are monitored by the computer system and these two factors become the inputs of the fuzzy controller. Their values are located onto the corresponding membership function to be fuzzified. The inference mechanism together with the rule base determines the fuzzy output values located onto the output membership function. The defuzzification stage defuzzies these values of the output membership function to get the output with real world constraint. The outputs from the fuzzy controller are the pulse width modulation (PWM) pulses. The PWM pulses are same in period but the duration of High represents the heating rate: the longer the duration of High, the more is the heating rate. The PWM driver circuit accepts these PWM pulsesand drives the dryer of the system with the respective heating rate.

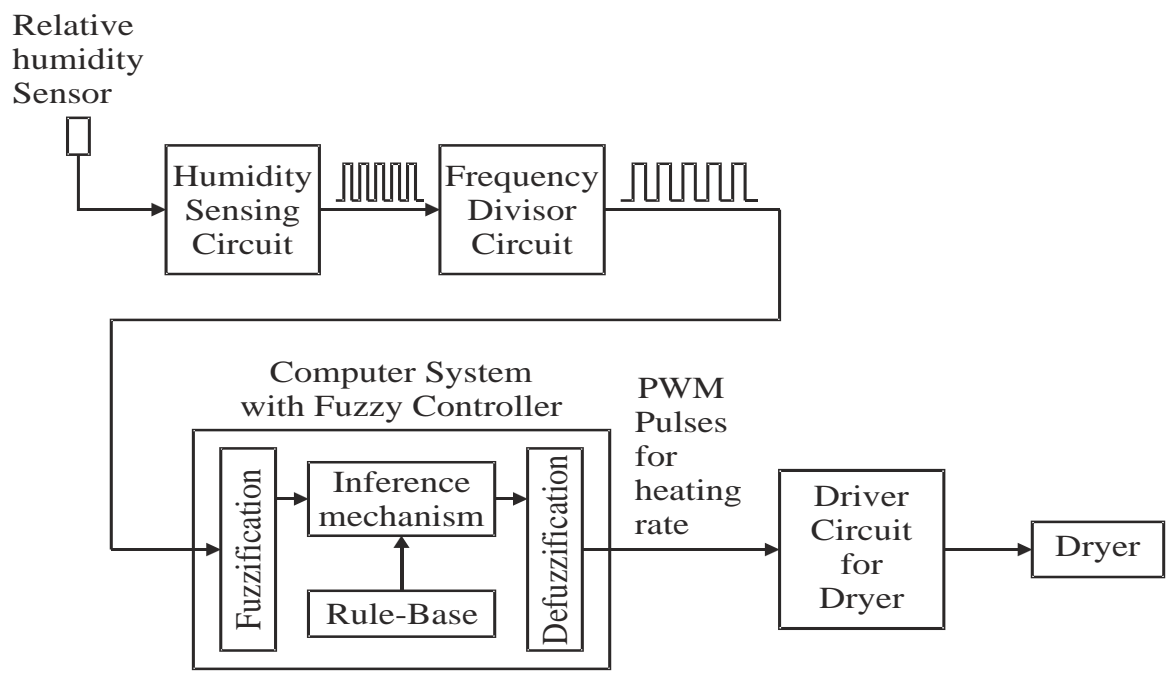

Figure5. Block Diagram of Fuzzy Logic Dryer Controller 


\subsection{Explanation of the System}

The relative humidity sensor changes its capacitance with the humidity. The result from the microcontroller which is to input the encoded 3-bit code representing the amount of humidity are applied into the computer system using the ACK, PAP and ONIOFF pins of the status register. To apply fuzzy pulses into the dryer fan driver circuit, the D0 of the data register of the LPT port is used [2]. All input and output operations to and from the computer system are carried out by means of opto-coupler isolation [1]. As the condition of moisture and its rate of change are 1 and 3 respectively, the fuzzy output, the duration of pulse, can be computed as follows. First of all, the two voltages are fuzzified to get the corresponding fuzzy values with respect to the membership functions concerned. Then according to the rule base (FAM table) together with the inference mechanism (MaxMin), output membership function together with the output fuzzy value, is obtained. This output is defuzzified and the direction and duration of pulse to drive the DC motor is achieved [1]. Test driving of the DC motor finds out the best-fit pulse width for different fuzzy outputs of the fuzzy controller are summarized in Table 5. There are six different fuzzy output values. As the fuzzy output becomes larger and larger, the width of the active pulse must also become longer and longer. The period of a cycle is taken as $150 \mathrm{~ms}$.

Table5. Fuzzy Outputs and Corresponding Pulse Widths

\begin{tabular}{|l|l|l|l|l|l|}
\hline $\begin{array}{l}\text { Fuzzy } \\
\text { Output }\end{array}$ & $\begin{array}{l}\text { Active } \\
\text { Width } \\
(\mathbf{m s})\end{array}$ & $\begin{array}{l}\text { Inactive } \\
\text { Width } \\
(\mathbf{m s})\end{array}$ & Direction & Duty Cycle & Waveform \\
\hline 0 & 30 & 120 & Alternate & $20 \%$ & $\square$ \\
\hline 1 & 50 & 100 & Up to the sign bit & $33 \%$ & $\square$ \\
\hline 1.57 & 78.5 & 71.6 & Up to the sign bit & $52 \%$ & $\square$ \\
\hline 2.33 & 116.5 & 33.5 & Up to the sign bit & $77 \%$ & $\square$ \\
\hline 2.6 & 130 & 20 & Up to the sign bit & $86 \%$ & $\square$ \\
\hline 3 & 150 & 0 & Up to the sign bit & $100 \%$ & \\
\hline
\end{tabular}

\subsection{Parallel Port Utilization of the System}

In this system the status pins of the LPT port are used to input the encoded 3 bits representing the amount of humidity into the computer system. Therefore, there are totally three bits to be input into the computer system. The data pins of the LPT port are used to output the fuzzy pulses to drive the dryer fan driver circuit [2]. Therefore, there is one output bit from the computer system. All input and output operations to and from the computer system are carried out by means of opto- coupler isolation as shown in Figure 6 and Figure 7.
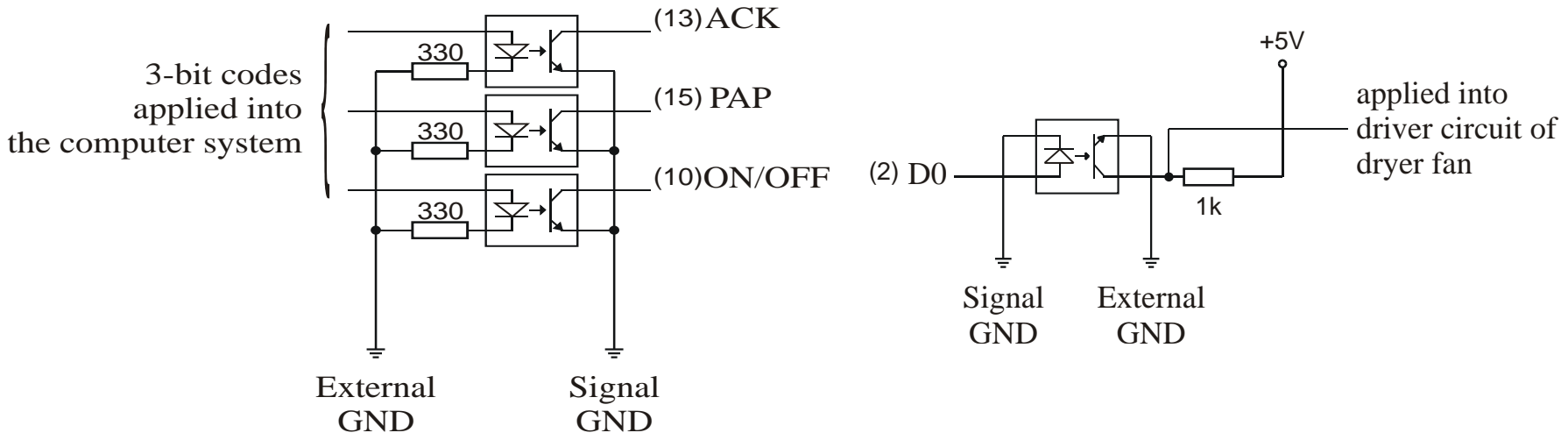

Figure6. Input Circuit to the Computer System.

Figure7. Output Circuit from the Computer System

To input the encoded 3-bit code representing the amount of humidity are applied into the computer system using the ACK, PAP and ON\OFF pins of the status register. To apply fuzzy pulses into the dryer fan driver circuit, the D0 of the data register of the LPT port is used. The data, status and control register utilization of the system is illustrated in Table 6. 
International Journal of Advances in Scientific Research and Engineering (ijasre), Vol 6 (4), April -2020

Table6. Data, Status and Control Register Utilization of the System

\begin{tabular}{|l|l|l|l|l|l|l|l|l|l|}
\hline \multirow{2}{*}{ Register } & \multirow{2}{*}{$\begin{array}{l}\text { I/O } \\
\text { Address }\end{array}$} & 7 & 6 & 5 & 4 & 3 & 2 & 1 & 0 \\
\hline Data & $0378 \mathrm{~h}$ & D7 & D6 & D5 & D4 & D3 & D2 & D1 & D0 \\
\hline \multirow{2}{*}{ Status } & $0379 \mathrm{~h}$ & BSY & ACK & PAP & $\begin{array}{l}\text { ON/ } \\
\text { OFF }\end{array}$ & ERR & X & X & X \\
\hline & & - & - & - & - & - & - & - & Pulse \\
\hline
\end{tabular}
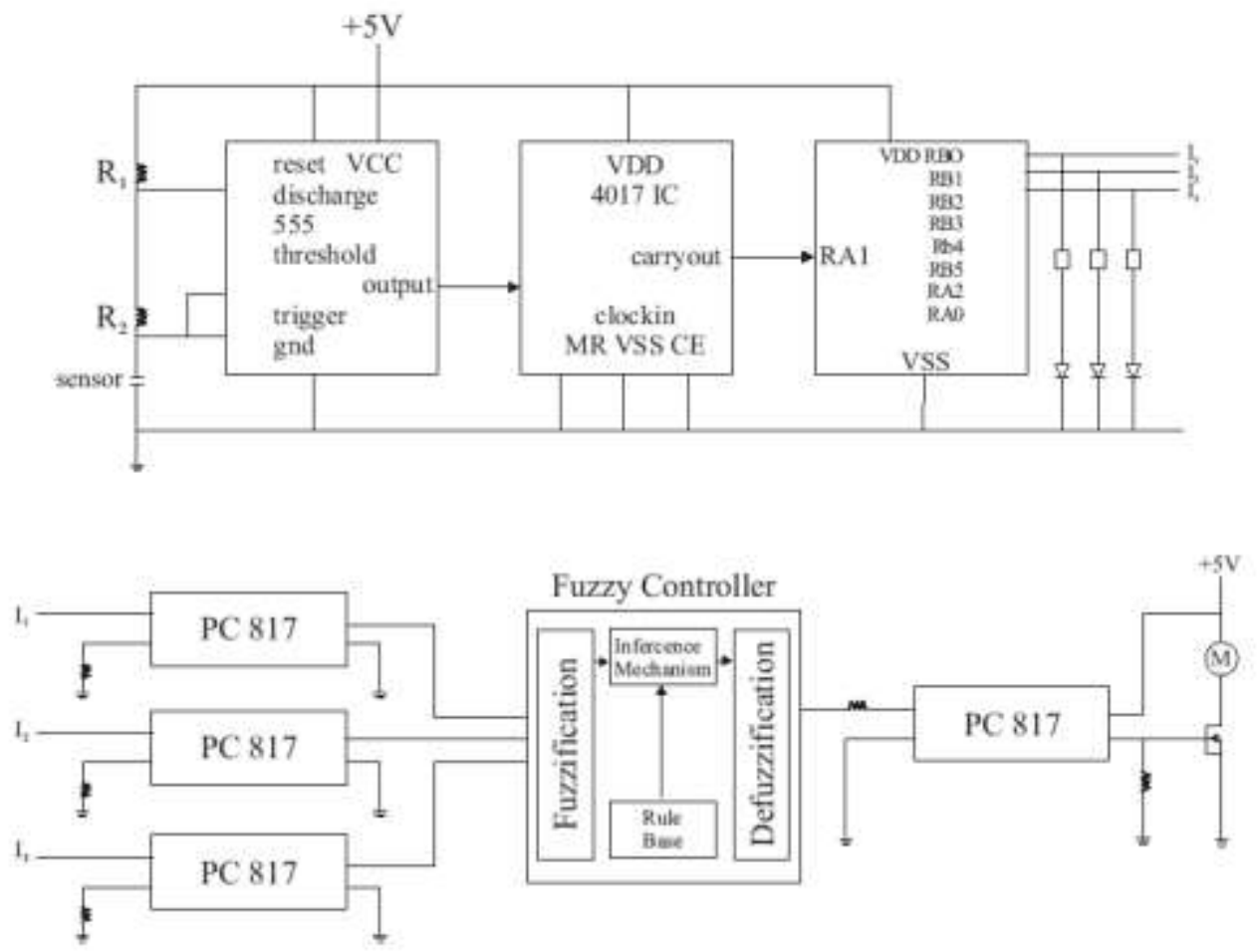

Figure8. Complete Circuit Diagram of the System

\section{CONCLUSION AND FURTHER EXTENSIONS}

This system is developed a moisture control system using fuzzy logic system to control the heating rate. The objectives of the system are: to certainly reduce the manual efforts and to get a fuzzy logic control system of real-time processing. A fuzzy logic controller offers a good alternative for such complex systems and has been very popular recently. Fuzzy logic can help us make decisions when lack of specific facts or the knowledge is imprecise or far from complete. The fuzzy controller as an artificial decision maker operates in a closed-loop system in real time.

In further extensions of this system, there can be more than eight different values for moisture values and its rate of changes. These larger value levels can be partitioned into more membership functions and the fuzzy values assigned to them become unlimited. There may more than five membership functions of the duration of the pulse. In such systems, the more and more fuzzy values can be assigned to different durations partitioned into more and more output membership functions. The FAM table's entries, membership functions partitioning the output durations of the pulse can also be extended for the future analysis of the system. Such extended system can be said to have more fuzzy features. DC motor used to blow the heater must be a heavy duty, high toque DC motors. The pulse width modulation (PWM) DC motor driver circuit must also be adapted to be suited with such DC motors.

\section{REFERENCES}


International Journal of Advances in Scientific Research and Engineering (ijasre), Vol 6 (4), April -2020

1. Floyd, Thomas.L, Digital Fundamental ( $8^{\mathrm{TH}}$ EDITION), Peasrson Education

2. Jan Axelson, Programming, Interfacing \& Using The PC's Parallel Printer Port, ISBN 0-9650819-1-5

3. Jame, J, Buckley and Esfandia Eslami, An Introduction To Fuzzy Logic And Fuzzy Set 2002, ISBN 3-7908-1447-4.

4. K.M.Passino and S.Yukovich, Fuzzy Control, Department of Electrical Engineering the Ohio State University.

5. Lee C.C., Fuzzy Logic Control System: Fuzzy Logic Controller Parts 1\&2, IEEE Transaction on Systems, Man and Cybernetics, vol.SMC-20, No-2, PP.404-435, March/April 1990.

6. L. Pirrello, L. Yliniemi, K. Leiviskä and M. Galluzzo, Self-Tuning Fuzzy Control Of A Rotary Dryer, 15th Triennial World Congress, Barcelona, Spain

7. L.W. Tan, F.S. Taip, N. Abdul Aziz, Simulation and Control of Spray Drying Using Nozzle Atomizer Spray Dryer, International Journal of Engineering \& Technology IJET Vol: 9 No: 10 